\title{
Demografische Entwicklung contra Sozialstaat? Eine ökonomische Potentialanalyse
}

\section{Norbert Reuter*}

\section{Dimensionen des Problems}

In der Öffentlichkeit gleichen Nachrichten über die Bevölkerungsentwicklung in der Regel Hiobsbotschaften. Der Tenor ist, dass

»die Alterung und die Abnahme der Bevölkerung in Deutschland [...] schwerwiegende Auswirkungen auf die Gesellschaft und die Funktionsfähigkeit der Sozialversicherungen haben ${ }^{\mathrm{I}}$ werden.

An besonders herausragender Stelle nutzt die von den Arbeitgeberverbänden finanzierte Initiative Neue Soziale Marktwirtschaft das Demografieargument, um die bestehenden sozialen Sicherungssysteme als prinzipiell zukunftsuntauglich zu klassifizieren und für einen Umstieg in die Privatisierung von Lebensrisiken zu werben. ${ }^{2}$

Belegt werden derartige Folgerungen mit der Veränderung des so genannten »Altenquotienten«. Er gibt das Verhältnis der Personen im Rentenalter zu Personen im erwerbsfähigen Alter an. Allerdings sind Altenquotienten alles andere als ein objektiver und wertfreier Maßstab. Es lassen sich für identische Zeiträume ganz unterschiedliche Quotienten bzw. Verhältnisse berechnen. Im Einzelnen hängt der Quotient unter anderem ab

- $\quad$ von der angenommenen Entwicklung der Lebenserwartung;

- $\quad$ von der angenommenen Entwicklung der Geburtenrate;

- $\quad$ von der angenommenen Höhe des Saldos aus Zu- und Abwanderung;

- $\quad$ von der Definition des Erwerbstätigenpotentials bzw. des Erwerbsalters (z.B. alle I5- bis 65-Jährigen oder nur alle 20- bis 60-Jährigen);

- $\quad$ von den Annahmen über den Eintritt ins Rentenalter (z.B. 6o Jahre oder 65 Jahre).

\section{Altenquotienten - untauglich zur Beschreibung realer Probleme}

Altenquotienten beschreiben also lediglich auf der Grundlage von verschiedensten Annahmen das Verhältnis des wie auch immer definierten Erwerbstätigenpotentials zur Anzahl der Rentnerinnen und Rentner. Neben großen Unsicherheiten über den tatsächlichen künftigen Bevölkerungsverlauf bleiben drei entscheidende Dinge ausgeblendet:

I. Hinsichtlich der gesamten wirtschaftlichen und sozialen »Belastung» einer Gesellschaft werden durch Altenquotienten lediglich Teilprobleme beschrieben. Entschei-

* Privatdozent, RWTH Aachen, ver.di Bundesvorstand, Bereich Wirtschaftspolitik, Berlin.

I So - als ein Beispiel unter vielen - zu lesen in der Frankfurter Allgemeine Zeitung vom 7.6.2003.

2 Nachzulesen auf der Homepage der Initiative: www.chancenfueralle.de. 
dend ist das Verhältnis der Erwerbstätigen zu allen Nicht-Erwerbstätigen. Zu letzteren gehören aber nicht nur die Menschen im Rentenalter, sondern auch diejenigen im noch nicht erwerbsfähigen Alter, also Kinder und Jugendliche. Eine alternde Gesellschaft zeichnet sich dadurch aus, dass zwar mehr Rentnerinnen und Rentner aus dem Sozialprodukt zu versorgen sind, dafür aber auch weniger Kinder und Jugendliche. Insofern stehen zusätzlichen »Belastungen« auch »Entlastungen« gegenüber. Dies wird im so genannten "Gesamtquotienten « zum Ausdruck gebracht, der das Verhältnis von Erwerbstätigen zu allen Nichterwerbstätigen (z. B. Personen über 65 und unter I5 Jahren) beschreibt.

Frei werdende finanzielle und personelle Ressourcen könnten so genutzt werden, die gesellschaftlichen Folgen einer zunehmenden Zahl alter Menschen zu mildern. Diese Entlastungswirkungen, die durch reine Altersquotienten nicht erfasst werden können, sind angesichts der derzeitigen Verhältnisse allerdings eher hypothetischer Natur. Das Problem ist nämlich, dass gegenwärtig erhebliche Versorgungsengpässe im Bereich Kinder und Jugendliche bestehen. Dies zeigt sich in fehlenden Kindertagesstätten-Plätzen über immer noch zu große Schulklassen bis hin zu dringend renovierungsbedürftigen Schulen. Insofern ist es angebracht, trotz einer sinkenden Zahl von Kindern und Jugendlichen in Zukunft finanzielle und personelle Ressourcen aus diesem Bereich nicht abzuziehen, sondern für eine materielle und personelle Besserversorgung zu nutzen. Dies könnte ein entscheidender Faktor dafür sein, dass in Deutschland auch wieder mehr Kinder als derzeit erwartet geboren würden. Mittelfristig könnte auf diese Weise die demografische Entwicklung ganz anders als heute erkennbar aussehen.

2. Um Auskunft über die tatsächliche »Belastung« einer Gesellschaft durch die demografische Entwicklung zu erhalten, ist der Gesamtquotient ohnehin weitgehend aussagelos. Entscheidend ist bei Alten- wie bei Gesamtquotienten nicht, wie viele Menschen insgesamt erwerbsfähig sind, einzig entscheidend ist, wie viele tatsächlich erwerbstätig sind. Eine in der Zukunft absolut und besonders im Verhältnis zu den Rentnerinnen und Rentnern sinkende Zahl von Erwerbsfähigen kann nicht beklagt werden, wenn heute Millionen von Menschen im Erwerbsalter arbeiten wollen, aber keine Möglichkeit hierzu haben. Bevor also eine steigende "Altenlast « in der Zukunft beklagt wird, muss erst einmal gefragt werden, welche Auswirkungen die bestehende Massenarbeitslosigkeit hat.

3. Auch wenn ein Abbau der Massenarbeitslosigkeit einen erheblichen Beitrag zur Lösung des Demografieproblems leisten kann, lässt sich hieraus die tatsächliche Belastung der Erwerbstätigen in der Zukunft noch nicht ableiten. Letztlich ausschlaggebend ist der Produktivitätsfortschritt. Er ermöglicht, dass die bzw. der einzelne Erwerbstätige mit gleichem Einsatz ein höheres Produktionsergebnis erzielen kann. Maßgeblich hierfür ist die technische und organisatorische Entwicklung. In der Vergangenheit hat dieser Faktor ganz wesentlich zur Wohlstandssteigerung beigetragen, und er wird seine Bedeutung auch in der Zukunft behalten. Insofern gibt erst die zusätzliche Berücksichtigung des Produktivitätsfaktors abschließend Auskunft über 
die tatsächliche Belastung der Erwerbstätigen durch die demografische Entwicklung in zehn, zwanzig, dreißig, vierzig Jahren.

\section{Die Konstruktion eines Problems - und zurück zur Realität ${ }^{3}$}

Auf den ersten Blick scheint der Anstieg der »Altenquotienten« die Dramatik der Entwicklung tatsächlich objektiv zu untermauern. Danach kommen heute auf eine Person im Rentenalter noch fast vier Personen im erwerbsfähigen Alter von I5 bis 65 Jahren. Dieses Verhältnis halbiert sich bis zum Jahre 2050 - je nach Annahmen einmal etwas mehr, einmal etwas weniger.

Allerdings sagen diese Relationen kaum etwas über die tatsächliche Belastung der Gesellschaft aus. Gegenwärtig ist bei weitem nicht jede/r zur Berechnung der Altersquotienten gezählte Erwerbsfähige auch erwerbstätig, was in Zeiten der Massenarbeitslosigkeit zu großen zahlenmäßigen Unterschieden führt. Heute sind nur knapp 69 Prozent der I5- bis 65-Jährigen beschäftigt. Somit stehen tatsächlich gegenwärtig nur 2,7 Erwerbstätige einer Person im Rentenalter gegenüber - bereits ein erheblicher Unterschied zu dem immer wieder genannten Verhältnis 4:I. Von einer zukünftigen Verdoppelung der »Alterslast« kann mit Blick auf die bereits heute bestehenden Verhältnisse nicht mehr gesprochen werden.

Welche Auswirkung hat nun ein Abbau der Arbeitslosigkeit auf das Verhältnis von Erwerbstätigen zu Rentnerinnen und Rentnern? Unterstellt, dass alle Personen, die im Jahr 2003 Erwerbsarbeit hatten oder aufnehmen wollten, einen Arbeitsplatz gehabt hätten (= Erwerbstätige [38,3 Millionen] + registrierte Arbeitslose [4,4 Millionen] + verdeckte Arbeitslose $^{4}$ [I,6 Millionen] + Stille Reserve [I,9 Millionen]), hätte die Zahl der Erwerbstätigen um 7,9 Millionen höher gelegen. Statt 38,3 Millionen hätten dann 46,2 Millionen Menschen gearbeitet. Es hätten dann nicht mehr nur 69 Prozent der 15- bis 65-Jährigen gearbeitet, sondern rund 83 Prozent.

Ein Abbau der Arbeitslosigkeit bis 20 oo würde bedeuten, dass sich das Verhältnis von Rentnerinnen und Rentnern zu Erwerbstätigen trotz anhaltender Alterung der Gesellschaft von heute I:2,7 auf I:2,8 zunächst verbessern (!) würde. Erst 2015 würde das Verhältnis dasjenige des Jahres 2003 unterschreiten. Im Jahr 2020 läge es unwesentlich schlechter bei I:2,5 und 2030 bei I:I,9. Bis 2050 würde es nur noch leicht auf I:I,6 zurückgehen (Abbildung I,

3 Für die folgenden Berechnungen wurde die mittlere (5.) Variante der Io. koordinierten Bevölkerungsvorausberechnung der Statistischen Bundesamtes zugrunde gelegt (dauerhaft I, 4 Kinder pro Frau, mittlerer Anstieg der Lebenserwartung, mittlerer Wanderungsüberschuss von jährlich 200.000 Personen) (vgl. Statistisches Bundesamt 2003).

4 Die verdeckte Arbeitslosigkeit errechnet sich als Arbeitslosenäquivalent aus Kurzarbeiterinnen und Kurzarbeitern, Teilnehmerinnen und Teilnehmern an Maßnahmen der aktiven Arbeitsmarktpolitik (ABM, SAM), Vollzeitteilnehmerinnen und -teilnehmer an beruflicher Weiterbildung und Personen im vorzeitigen Ruhestand. Die Zahl ergibt sich hier aber ohne ABM und SAM, da diese bereits als Erwerbstätige gezählt sind.

5 Zur Stillen Reserve zählen diejenigen Personen, die bei ungünstiger Arbeitsmarktlage die Arbeitssuche entmutigt aufgeben, aber bei besserer Lage wieder Arbeitsplätze nachfragen würden. 
s.u.). Damit ist man von einer Verdoppelung der Belastung der Erwerbstätigen bis 2050, wie es der Altenquotient ohne Berücksichtigung des Arbeitslosenproblems suggeriert, einmal mehr weit entfernt. Danach entschärft sich die Lage aller Voraussicht nach ohnehin kontinuierlich, da dann die geburtenschwachen Jahrgänge das Rentenalter erreichen werden. Der Bevölkerungspilz, der sich aus der Bevölkerungstanne herausgebildet hat, wird dann zunehmend die Form einer schlankeren Bevölkerungssäule annehmen.

\section{Abbildung I: Alters-/Erwerbstätigenquotienten bei Abbau der Arbeitslosigkeit*}

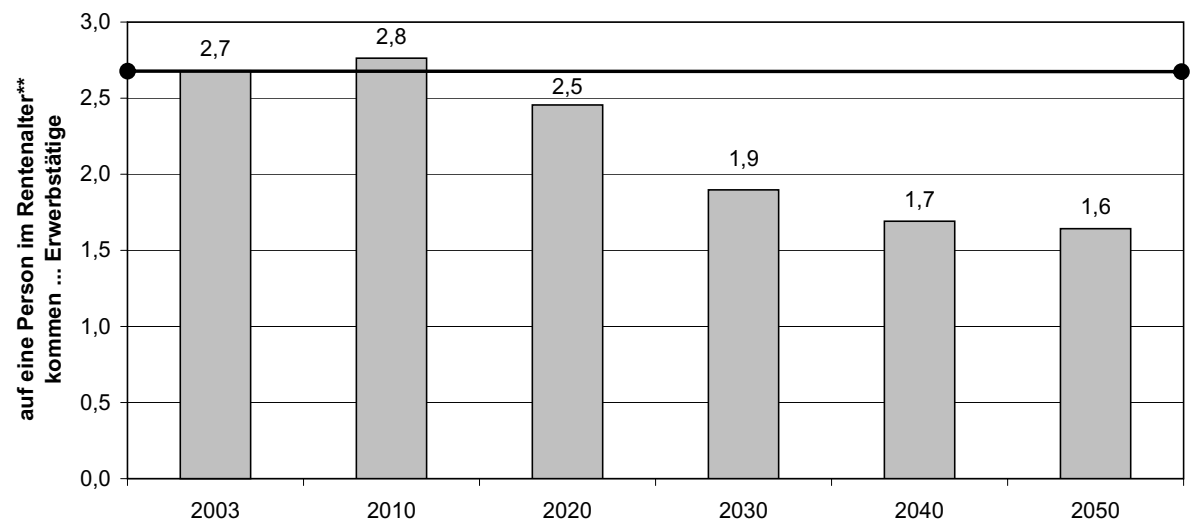

* Unterstellt ist, dass 83\% der I5- bis 65-jährigen ab 2010 arbeiten. Dies entspricht der Absicht der I5- bis 65-jährigen des Jahres 2003 (= Erwerbstätige + registrierte Arbeitslose + verdeckte Arbeitslose + stille Reserve).

** Personen über 65 Jahren.

Quelle: Statistisches Bundesamt 2003

\section{Von Pro-Kopf-Verhältnissen zur Produktivitätsanalyse}

Mit Blick auf die zahlenmäßigen Verschiebungen zwischen der Gruppe der »Jungen « und der »Alten« zeigt sich, dass nicht die Alterung der Gesellschaft, sondern die Arbeitslosigkeit das größere Problem ist. Durch deren Abbau würden demografische Effekte erst nach 2015 zu einem ungünstigeren Pro-Kopf-Verhältnis im Vergleich zum aktuellen Wert führen. Doch auch danach wird es zu keiner erhöhten Belastung der Gesellschaft durch die Alterung der Gesellschaft kommen. Dies ist dem Produktivitätsfortschritt zu verdanken. Er ermöglicht, dass in der Zukunft in der gleichen Zeit ein höheres Produktionsergebnis je erwerbstätige Person erzielt wird. Die Produktivität gibt somit Auskunft über die Effizienz der Produktion in einer Volkswirtschaft.

Der Steigerung der Produktivität ist es im Wesentlichen zu verdanken, dass die demografische Entwicklung bereits in der Vergangenheit ohne größere soziale Verwerfungen ablaufen konnte. Sie sichert auch in Zukunft, dass steigende Einkommen und steigende Renten durch ein wachsendes Sozialprodukt pro Kopf der Bevölkerung erwirtschaftet wer- 
den können, ohne dass es zu realen Mehrbelastungen der Erwerbstätigen kommt. Vergleicht man das Jahr 2003 mit dem Jahr 1960, so zeigt sich, dass z. B. in den alten Bundesländern mit nur noch gut 8o Prozent der gesamten Arbeitsstunden (Arbeitsvolumen) des Jahres I960 ein real mehr als dreifach so hohes Bruttoinlandsprodukt (BIP) geschaffen wird.

Da ein Ende der Produktivitätsentwicklung nicht in Sicht ist - die von der Regierung eingesetzte »Rürup-Kommission« unterstellt ebenso wie die Prognos AG bis zum Jahr 2040 ein Produktivitätswachstum von durchschnittlich I,8 Prozent pro Jahr (Bundesministerium für Gesundheit und Soziale Sicherung 2003: 6I) -, wird es möglich, auch bei einem sich verschlechternden Pro-Kopf-Verhältnis zwischen Erwerbstätigen einerseits und Rentnerinnen und Rentnern andererseits steigende Sozialleistungen zu finanzieren, ohne dass die Erwerbstätigen real schlechter gestellt werden. Dies lässt sich wiederum anhand der jüngsten amtlichen Prognose über die Bevölkerungsentwicklung zeigen. Gerechnet wurde wieder mit der mittleren (5.) Variante und in Preisen von 2003.

Im Jahr 2003 haben die 38,3 Millionen Erwerbstätigen in Deutschland ein BIP von 2,I Billionen $€$ erwirtschaftet. Dies entspricht bei 82,5 Millionen Einwohnerinnen und Einwohnern einem Pro-Kopf-Wert von $25.760 €$ (Abbildung 2, S. 28). Die abnehmende Zahl der Personen im erwerbsfähigen Alter führte nur dann zu einem sinkenden BIP pro Kopf, wenn die Arbeitslosigkeit bis 2050 auf dem heutigen Niveau verharrte und eine Stagnation der Produktivität unterstellt wird (linke Säulen). Unter dieser völlig unrealistischen Annahme würde das Pro-Kopf-BIP bis 2050 um I3 Prozent auf nur noch rund 22.400 € sinken. Dann hieße es in der Tat: „Gürtel-enger-schnallen«.

Aber bereits bei einem unterstellten Produktivitätsfortschritt von nur I,5 Prozent pro Jahr und erwerbstätiger Person ${ }^{6}$ sieht die Entwicklung ganz anders aus - selbst wenn man annimmt, dass keinerlei Erfolge bei der Bekämpfung der Arbeitslosigkeit gemacht würden. Dann würden auch in Zukunft wie im Jahr 2003 nur 69 Prozent der 15- bis 65Jährigen Erwerbsarbeit leisten können (mittlere Säulen). Die Anzahl der Erwerbstätigen würde dann von heute 38,3 Millionen auf 30,3 Millionen im Jahr 2050 sinken. Dennoch würde das von ihnen erwirtschaftete BIP pro Kopf der Bevölkerung bis 2050 dank der steigenden Produktivität um insgesamt gut 75 Prozent auf $45.150 €$ steigen.

Unterstellt man dann noch Erfolge bei der Bekämpfung der Arbeitslosigkeit - die Zahl der Erwerbstätigen würde bis 2050 trotz Bevölkerungsrückgang statt auf 30,3 Millionen nur auf 36,5 Millionen sinken -, steigt das Inlandsprodukt sogar um III Prozent auf $54.470 €$ pro Kopf im Jahr 2050 (rechte Säulen). Trotz der Alterung der Gesellschaft und des damit verbundenen Rückgangs der Erwerbstätigen um knapp fünf Prozent würde sich das BIP also mehr als verdoppeln. (Abbildung 2, S. 28)

Damit lässt sich festhalten: Das BIP pro Kopf wird im Jahre 2050 unter der realistischen Annahme eines Produktivitätsfortschritts von I,5 Prozent im ungünstigsten Fall (die Arbeitslosigkeit bleibt konstant hoch) um 75 Prozent und im günstigsten Fall (die Arbeitslosigkeit verschwindet) um III Prozent höher als heute liegen.

6 In den I980er Jahren betrug der Produktivitätsfortschritt pro erwerbstätiger Person und Jahr I,7 Prozent, in den I99oer Jahren waren es I,3 Prozent. 
Abbildung 2: Produktivitätsfortschritt schlägt Alterung ${ }^{I}$

Reales Bruttoinlandsprodukt pro Kopf mit ${ }^{2}$ und ohne Arbeitslosigkeit

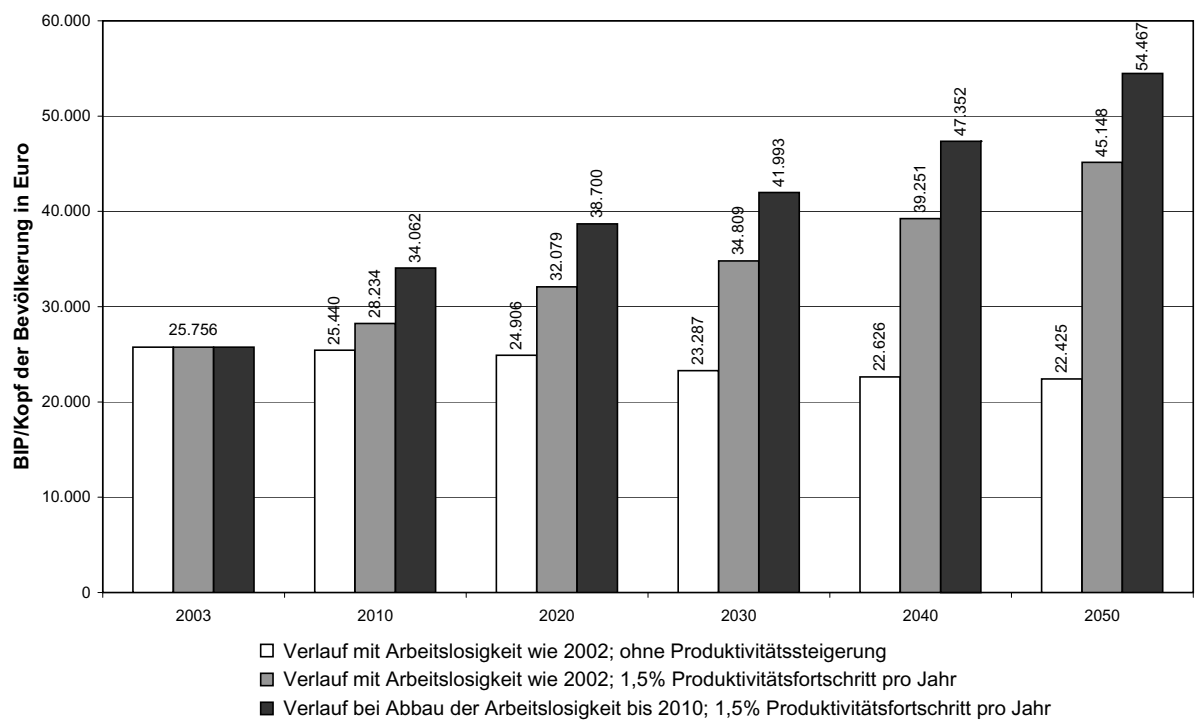

I Grundlage der Berechnungen ist die mittlere (5.) Variante der amtlichen Bevölkerungsprognose.

2 Unterstellt ist, dass bis 2050 derselbe Anteil der 15- bis 65-jährigen (= 69 Prozent) arbeitet wie 2003.

3 Unterstellt ist, dass ab 201083 \% der I5- bis 65-jährigen arbeiten; dies entspricht der Absicht der I5- bis 65-jährigen des Jahres 2003 (= Erwerbstätige + registrierte Arbeitslose + verdeckt Arbeitslose + Stille Reserve).

\section{Quelle: Statistisches Bundesamt 2003}

Diese Erhöhung der jährlichen Wertschöpfung ermöglicht es jeder Bürgerin und jedem Bürger, trotz der Alterung unserer Gesellschaft in den nächsten Jahrzehnten durchgehend auch Einkommenssteigerungen zu erhalten. Das heißt, die Spielräume zur Finanzierung des Sozialstaats werden in Wirklichkeit nicht kleiner, sondern erweitern sich weiterhin!

Selbst wenn bis 2050 keine Fortschritte beim Abbau der Arbeitslosigkeit in Deutschland gemacht würden, ermöglichte der Anstieg des BIP auf $45.150 €$ bis zum Jahr 2050 Einkommenssteigerungen von real I,2 Prozent pro Jahr für jede Bürgerin und jeden Bürger. Bei einem Abbau der Arbeitslosigkeit würde sich dieser Wert sogar auf I,6 Prozent erhöhen - jeweils vorausgesetzt, der gesamte Einkommenszuwachs würde gleichmäßig auf alle Köpfe verteilt. Liegt der Produktivitätsfortschritt höher als die angenommenen I,5 Prozent pro Jahr, würden sich die Spielräume noch erheblich erweitern.

\section{Gerechte Verteilung von Arbeit und Einkommen als zentrales Problem}

Die vorangehenden Ausführungen weisen auf grundsätzlich mögliche Entwicklungspfade hin. Voraussetzung dafür, dass ein gesellschaftlich günstiger Entwicklungspfad auch Rea- 
lität wird, ist, dass die Verteilung der Produktivitätsgewinne in der Zukunft zwischen Jung und Alt, Arm und Reich sowie Arbeit und Kapital gleichmäßig geschieht. Wenn es einzelnen Gruppen dagegen gelingt, Erhöhungen des individuellen Einkommens zu realisieren, die oberhalb der genannten Steigerungsraten liegen, bedeutet dies automatisch, dass für andere entsprechend geringere Spielräume bleiben.

Befürchtungen dieser Art sind durchaus angebracht: Dies lässt sich aus der extrem ungleichen Verteilung des Geldvermögens folgern. Sie stellt einen guten Indikator für die Verteilungssituation eines Landes dar, da sich hier die voran gegangene Einkommensentwicklung als Folge nicht nur der Primär-, sondern auch der Sekundärverteilung niederschlägt.

\section{Abbildung 3: Privates Geldvermögen in Deutschland}

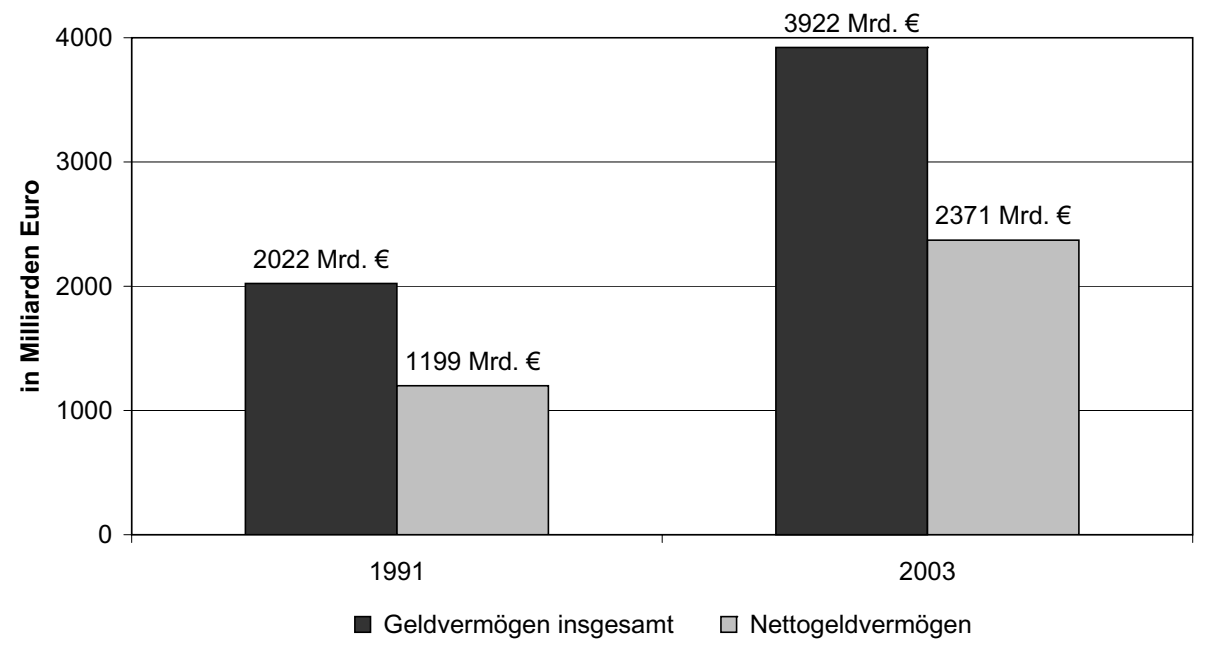

Quelle: Deutsche Bundesbank 2004: 54

Das jährlich erwirtschaftete BIP ist seit I99I nominal um 40 Prozent gestiegen. Im selben Zeitraum verdoppelte sich das gesamte nominale Nettogeldvermögen (= Bruttogeldvermögen abzüglich der Schulden) von I.I99 Milliarden $€$ auf 2.37I Milliarden $€$ (Abbildung 3). Das Hauptproblem ist, dass sich dieser enorme Zuwachs auf eine kleine, sehr reiche Schicht konzentriert: Nach der letzten bislang verfügbaren Verteilungserhebung entfielen 1998 über 50 Prozent des gesamten Nettogeldvermögens auf gerade einmal zehn Prozent der reichsten Haushalte, während die unteren 50 Prozent auf nicht einmal fünf Prozent des gesamten Nettogeldvermögens kamen. 1993 war die Ungleichverteilung noch etwas geringer. In diesem Jahr verfügten die reichsten zehn Prozent der Haushalte »erst» über gut 46 Prozent des Geldvermögens, während die unteren 50 Prozent immerhin noch fast acht Prozent des gesamten Geldvermögens besaßen (vgl. Sachverständigenrat 200o) (Abbildung 4, S. 30). 
Abbildung 4: Verteilung des Nettogeldvermögens 1993 und 1998 in Deutschland

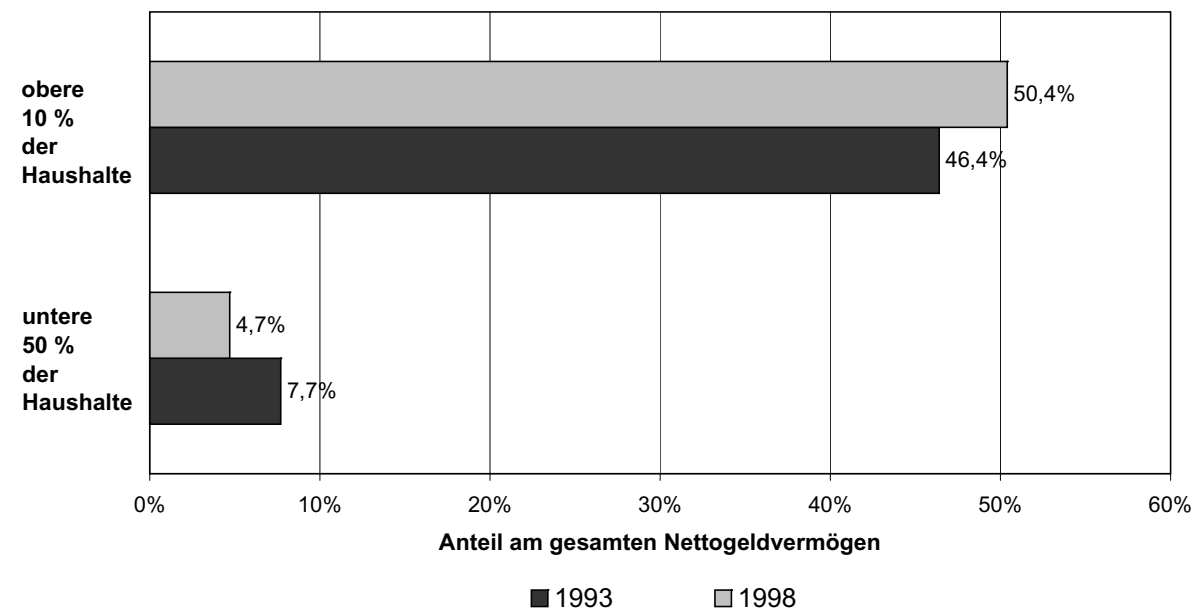

Quelle: Sachverständigenrat zur Begutachtung der gesamtwirtschaftlichen Entwicklung 2000: 267

\section{Wirtschaftspolitik gefordert}

Die Bevölkerungsstruktur in Deutschland hat sich in der Vergangenheit verändert und wird sich auch weiter verändern. Es ist jedoch ein Trugschluss, dass deshalb der Sozialstaat abgebaut werden muss. Demografische Argumente verschleiern lediglich reale wirtschaftsund sozialpolitische Probleme. Entscheidend für den Erhalt des Sozialstaats ist, dass die Früchte des Produktivitätsfortschritts allen zugute kommen. Erste Voraussetzung hierfür ist, dass die Umverteilung von Löhnen zu den Gewinnen und von unten nach oben beendet und umgekehrt wird. Bereits heute ist das Finanzierungsproblem bei den Sozialversicherungen keine Folge der Bevölkerungsentwicklung, sondern hauptsächlich der hohen Arbeitslosigkeit und der schwachen Lohnentwicklung der letzten Jahre. Der Abbau der Arbeitslosigkeit und ein Anstieg der Arbeitseinkommen im Ausmaß des verteilungsneutralen Spielraums (Produktivitätsfortschritt + Inflationsrate) würden die Probleme maßgeblich entschärfen. Beide Faktoren trügen dazu bei, dass die Einnahmen der Sozialversicherungen deutlich anstiegen.

Die Einbeziehung von Gewinn- und Vermögenseinkommen in das System der Sozialversicherungen würde das Finanzierungsproblem weiter entschärfen. Solange die Finanzierung des Sozialstaats primär aus dem Arbeitseinkommen erfolgt und Gewinn- und Vermögenseinkommen verschont bleiben, sind Verteilungsprobleme vorprogrammiert. Insofern muss die Finanzierung der Sicherungssysteme auf mehr Schultern verteilt, d.h. die Bemessungsgrundlage erweitert werden. Ansätze, auch Kapitaleinkommen sozialversicherungspflichtig zu machen, weisen in die richtige Richtung. Eine umfassende Ausweitung des Kreises der Beitragszahlerinnen und Beitragszahler trüge dem Grundgedanken von Sicherheit, Solidarität und Gerechtigkeit in einer veränderten Arbeitswelt Rechnung. 
Sollten sich dann in Zukunft noch Finanzierungslücken auftun, bliebe immer noch die Möglichkeit einer Erhöhung der Sozialversicherungsbeiträge. Bei einer erfolgreichen Tarifpolitik bedeutete dies keine erhöhte Mehrbelastung der Beschäftigten. Würde in Zukunft die Steuerlast wieder gerechter verteilt werden (z.B. durch Verzicht auf die Senkung des Spitzensteuersatzes und die Einführung einer Vermögensteuer), könnte ggf. auch die Möglichkeit höherer staatlicher Zuschüsse in Erwägung gezogen werden.

Gerade angesichts der demografischen Entwicklung wird damit eine Politik immer wichtiger, die die Herstellung einer gleichmäßigeren Verteilung von Einkommen und Vermögen zum Ziel hat. Grundsätzlich ermöglichen der bestehende gesellschaftliche Reichtum und sein weiterer Anstieg den Erhalt des Sozialstaats auf dem erreichten Niveau. Eins ist jedoch klar: Sofern die Polarisierung von Einkommen und Vermögen ungebremst voranschreitet und dieser Trend im Rahmen der Steuer- und Finanzpolitik sogar noch verstärkt wird, ist jede denkbare Bevölkerungsentwicklung mit wachsenden sozialen Problemen verbunden.

Die Lösung anstehender Probleme hängt letztlich davon ab, ob und inwieweit Staat und Gesellschaft in der Lage sind, den notwendigen sozialen Ausgleich in der Zukunft zu bewerkstelligen. Jeder Versuch, die anstehenden Probleme durch mehr Privatisierung und private Vorsorge zu lösen, ist demgegenüber zum Scheitern verurteilt. Die Privatisierung der sozialen Absicherung löst keine demografiebedingten Probleme, sondern verstärkt die Tendenz zur Polarisierung weiter (vgl. Reuter 2002). Der Teufel würde gewissermaßen mit dem Belzebub ausgetrieben.

\section{Fazit}

Die anhaltende Erhöhung der Zahl von Personen im Rentenalter ist keineswegs mit einer Lawine vergleichbar, die unaufhaltsam auf den Sozialstaat zurollt und ihn früher oder später niederwalzt, sofern es »Reformer« und »Modernisierer« nicht bereits vorher - mehr oder weniger geordnet - erledigt haben. Nicht Bevölkerungsverhältnisse entscheiden in erster Linie über die Belastungen des erwerbsfähigen Teils der Bevölkerung, sondern das Wachstum des Sozialprodukts, die Produktivität, die Entwicklung des Reallohns, die Beschäftigungshöhe, die Besteuerung von Kapital und Arbeit sowie die Verteilung des Sozialprodukts. Faktisch verhindert der beständige Hinweis auf die »demografische Krise« wichtige Reformen, die auf die Beseitigung der akuten sozialen Probleme zielen.

Der jährlich erzeugte sowie der schon geschaffene Reichtum und die Produktivitätsen twicklung sind ausreichend, um heutigen und künftigen demografischen Verschiebungen ohne höhere Belastung der Einzelnen zu begegnen. Akute Panik- und Untergangsstimmung ist fehl am Platz und dient in der Regel nur der Durchsetzung privatwirtschaftlicher Gewinninteressen - nicht zuletzt der privaten Versicherungswirtschaft.

Wie andere Länder auch steht Deutschland vor der Herausforderung, auf die Bevölkerungsentwicklung im demokratischen Prozess verteilungspolitisch reagieren zu müssen. Nicht zuletzt deshalb sind eine Verbreiterung und damit Stärkung der Finanzierungsbasis des Sozialstaats auf der einen sowie eine Investitions-, Beschäftigungs-, Verteilungs- und Bildungsoffensive auf der anderen Seite notwendiger denn je. 


\section{Literatur}

Bundesministerium für Gesundheit und Soziale Sicherung (Hg.) (2003): Nachhaltigkeit in der Finanzierung der sozialen Sicherungssysteme. Bericht der Kommission, Berlin

Deutsche Bundesbank (2004): Die gesamtwirtschaftlichen Finanzierungsströme im Jahr 2003, in: Deutsche Bundesbank Monatsbericht, Jg. 56, H. 6, S. 45-60

Reuter, Norbert (2002): Generationengerechtigkeit als Richtschnur der Wirtschaftspolitik?, in: Butterwegge, Christoph/Klundt, Michael (Hg.), Kinderarmut und Generatione ngerechtigkeit. Familien- und Sozialpolitik im demographischen Wandel, Opladen, S. $8 \mathrm{I}-98$

Sachverständigenrat zur Begutachtung der gesamtwirtschaftlichen Entwicklung (2000): Jahresgutachten 2000/or. Chancen auf einen höheren Wachstumspfad, Wiesbaden

Statistisches Bundesamt (2003): Bevölkerung Deutschlands bis 2050. Io. koordinierte Bevölkerungsvorausberechnung, Wiesbaden

\section{Besser als sein Ruf: Der Standort Deutschland und die Rückkehrer Anja Schulz*}

\section{Die Standortdebatte}

Laut aktueller Meinungsumfragen unter deutschen Managerinnen und Managern steht es um den »Standort Deutschland « schlecht. 74 Prozent der vom Hamburger PsephosInstitut befragten Führungskräfte bewerten heute die heimischen Rahmenbedingungen für erfolgreiches wirtschaftliches Handeln negativ. Besonders unzufrieden äußern sich die Unternehmensspitzen aus dem Baugewerbe und dem produzierenden Sektor (vgl. Schwarz 2004). Bezogen auf den Mittelstand stellt sich die Stimmung noch düsterer dar. Nach jüngsten Ergebnissen des Instituts für Mittelstandsforschung (IfM) Bonn erwartet jeder vierte Unternehmer aus diesem Sektor »eine Verschlechterung der Lage« (IfM 2004: 22). Der Mittelstandsmonitor der Kreditanstalt für Wiederaufbau (KfW) 2004 und das Mittelstandsbarometer 2004 bestätigen dies (KfW 2004: I3, Müller et al. 2004: IO). Als Nachteile des Standort Deutschland gelten immer wieder die hohen Lohn(neben)kosten, bürokratische Hemmnisse, die hohe Steuerbelastung, der unflexible Arbeitsmarkt sowie das deutsche Arbeitsrecht (vgl. z.B. o.V. 2002a). "Steuerlast, Arbeitskosten und Bürokratie treiben Unternehmen aus Deutschland hinaus«, heißt es auch plakativ (o.V. 2002b).

* Universität Dortmund, Lehrstuhl für Unternehmensführung. 